En los Estudios de Traducción, suele tomarse al estadounidense ARTICULOS · 183-197 Lawrence Venuti como el traductólogo más representativo del método de extranjerización. Sin embargo, son pocos los que saben que China cuenta también con un gran teórico de la traducción y traductor profesional, Lu Xun, quien elaboró su propia teoría de extranjerización muchos años antes que Venuti. En este artículo nos proponemos hacer un análisis contrastivo de las teorías de traducción de Lu Xun y Venuti, centrándonos en particular en el método de la extranjerización, que ilustraremos con ejemplos prácticos a partir de sus propias traducciones. Tras realizar una comparación detallada entre estos dos autores, llegamos a la conclusión de que sus visiones acerca de la extranjerización comparten muchos puntos en común, pero también muestran algunas diferencias, inevitables debido a los contextos históricos y sociales de cada uno.

PALABRAS CLAVE: Lawrence Venuti, Lu Xun, apropiación, extranjerización.

\title{
La extranjerización como método traductor: coincidencias y divergencias entre Lu Xun y Venuti
}

\section{Helena CASAS-Tost ${ }^{\mathrm{y}}$ Y Niu Ling Universidad Autónoma de Barcelona}

I Adscrita al grupo de investigación TXICC, CERAO y al grupo de investigación Interasia.

Esta investigación se inscribe en el marco del programa de SGR (IIO3-2009) de la Generalitat de Catalunya y forma parte del proyecto «El impacto de Asia Oriental en el contexto español: producción cultural, política(s) y sociedad» (referencia FFI2OII29090/FISO).

\section{Foreignisation as a translation method: similarities and differences between Lu Xun and Venuti}

In the circle of translation studies, Lawrence Venuti is usually considered the most prominent representative of the method of foreignization. However, very few people know that in China there is also a great theorist and translator, Lu Xun, who developed his own method of foreignization years earlier than Venuti. This article aims at carrying out a contrastive analysis of Lu Xun's and Venuti's translation theory. It will mainly focus on the method of foreignization, which will be also illustrated with examples of their translations. After a detailed comparison between the two authors, we can conclude that their views on foreignization present many similarities, but also some differences, due to their different historical and social contexts.

KEY WORDS: Lawrence Venuti, Lu Xun, domestication, foreignization. 


\section{INTRODUCCIÓN}

Los conceptos de apropiación y extranjerización 184 son recurrentes en la historia de la teoría y la práctica de la traducción. Aunque han sido tratados adoptando diferente terminología y desde varias ópticas dentro de la Traductología (traducción libre versus literal, equivalencia formal versus dinámica, etc.), son términos que están inevitablemente unidos a un nombre propio en Occidente: el del traductólogo estadounidense Lawrence Venuti, quien propuso en una de sus obras más citadas y conocidas, The Translator's Invisibility - A History of Translation (1995), los términos domestication and foreignization en su sentido moderno.

No obstante, estos conceptos - y su carga ideológica correspondiente- fueron ya empleados anteriormente en China por Lu Xun (鲁迅) (I88I-I936). A pesar de su gran influencia como traductor y teórico en China, lo cierto es que en Occidente son muy pocos los que lo conocen en cualquiera de sus vertientes, particularmente la de teórico de la traducción. Si bien en China sí se conocen las teorías de traducción occidentales, incluyendo los postulados de Venuti, Occidente prácticamente vive de espaldas a las teorías procedentes de otras zonas, incluso las formuladas por autores tan destacados en sus propias tradiciones como Lu Xun.

En este artículo nos proponemos hacer una revisión de los postulados de estos dos teóricos de la traducción acerca de un concepto que define y caracteriza a ambos: la extranjerización. Creemos que es muy interesante comprobar que, aún siendo de diferentes países y perteneciendo a periodos históricos distintos, sus ideas respecto a este método de traducción guardan muchos puntos en común y merecen un estudio en profundidad.

\section{LU XUN Y LA EXTRANJERIZACIÓN}

Lu Xun está considerado el padre de la literatura moderna en China, siendo uno de los primeros escritores que empleó la lengua vernácula en substitución del chino clásico en las obras literarias. Como escritor, Lu Xun dejó una impronta indeleble en la literatura china y sus obras han sido traducidas a numerosas lenguas. Como traductor, su labor no fue menos importante, ya que fue muy prolífico y tradujo al chino a los grandes maestros de la literatura universal de hasta veinte países, incluyendo obras en inglés, japonés, ruso, etc. (Xia y Cha, 2004, citado en Tai, 2012: 160). Sobre la base de su extensa práctica y de su concepción de la traducción como herramienta al servicio del pueblo y de reforma social, Lu Xun expuso y llevó a la práctica sus postulados teóricos de la traducción, entre los que destaca sobre todo su aportación acerca de la noción de extranjerización, que veremos a continuación.

\section{I. Concepción de la extranjerización por parte de Lu Xun}

En el año 1935, Lu Xun expuso sus ideas sobre la extranjerización en traducción en el artículo 《“题未定” 草》（一至三） “ $T i$ wei ding” cao (yi zhi san) [Borrador de "Título aún no fijado" (número I a 3)]. Segú n Lu Xun (I980e: 352). Antes de empezar a traducir es preciso decidir si se debe hacer todo lo posible para domesticar el texto original o si se deben conservar los rasgos exóticos que pueda tener. En base a ello, distingue dos métodos de traducción: la apropiación (归化guibua) y la extranjerización (异国情调 yiguo qingdiao) ${ }^{2} \mathrm{y}$ se declaró partidario del segundo. Compara la

\footnotetext{
2 异国情调 (yiguo qingdiao) literalmente significa «toques exóticos de países extranjeros».
} 
traducción de una obra extranjera con un viaje a un lugar desconocido, de modo que ante una traducción considera que el lector debe experimentar las sensaciones que puede tener al viajar por países extranjeros, con sus toques exóticos y particulares. Para lograrlo opina que lo mejor es optar por la extranjerización.

Por otra parte, Lu Xun formaba parte de los intelectuales que abogaban por una reforma lingüística y literaria que según ellos contribuiría en gran medida a la modernización de China, un país que se encontraba en una situación crítica desde el punto de vista político, económico y también cultural, y que consideraban que necesitaba abrirse al mundo y aprender de las potencias extranjeras.

Aparte de estas ideas generales acerca de la traducción y de su papel en la modernización del chino, Lu Xun plantea unas estrategias concretas para llevar a cabo este método de traducción en diferentes niveles. En el plano léxico era partidario del uso de préstamos y calcos para traducir conceptos desconocidos, en vez de apropiarlos. En su opinión, al enfrentarse a nuevas palabras los lectores chinos podrían incrementar la riqueza expresiva y la precisión de su lengua e impulsar así el desarrollo lingüístico de su país. Para explicar mejor esta idea, Lu Xun (1980b: 382-383) puso el ejemplo de la palabra 罢工 (bagong), que significa «huelga» y que no existía antes en la lengua china hasta que fue introducida como un préstamo en el año 1925, y que se emplea con mucha frecuencia actualmente.

Esta idea la llevó al extremo de traducir también los nombres propios. Aunque hoy en día es muy común traducir fonéticamente los nombres propios, a principios del siglo $\mathrm{xx}$ los traductores chinos solían adaptarlos, lo cual tenía como objetivo facilitar la lectura y la memorización por parte de los lectores. Sin embargo, Lu Xun se declaró en contra de este tipo de traducción y defendió conservar el sonido original, sin modificar u omitir nada en los antropónimos y topónimos extranjeros, puesto que cualquier omisión o cambio sería considerado una infidelidad $(\mathrm{Lu}$ Xun, I980d: 157).

En cuanto al plano oracional, Lu Xun propuso conservar en la medida de lo posible las estructuras sintácticas originales, pese a las substanciales diferencias entre el chino y las lenguas occidentales. Mientras que en éstas suelen aparecer cláusulas compuestas muy largas, los chinos están acostumbrados a oraciones más bien cortas. Por ello, para expresar el sentido con mayor claridad y fluidez, muchos traductores chinos cambian el orden de las oraciones o las fragmentan en oraciones cortas añadiendo entre ellas explicaciones o nexos. Lu Xun, por el contrario, se propuso conservar las cláusulas compuestas de las lenguas extranjeras a costa, en cierta medida, de la fluidez. Esperaba que, con las traducciones extranjerizantes, el chino podría incorporar nuevos elementos sintácticos $y$, de esta forma, lograr un mejor desarrollo y modernización.

Para Lu Xun, la aportación de la extranjerización al progreso y evolución del japonés era sin duda aleccionadora para los chinos. Según él, el japonés moderno ha adoptado muchas estructuras sintácticas extranjeras a través de las traducciones extranjerizantes y, en comparación con el clásico, resulta más fácil de traducir a otras lenguas, sin que se pierda el tono del texto original (Lu Xun, I980a: 199).

Además, según Lu Xun, la extranjerización no debe limitarse sólo a la traducción de palabras u oraciones: ha de abarcar también los signos de puntuación. Para los chinos, los signos de puntuación son un fenómeno importado de Occidente, ya que en los textos chinos antiguos carecían de puntuación: sólo se indicaban unos puntos que marcaban una pausa para facilitar 
la comprensión de los lectores. Lu Xun propuso introducir los signos de puntuación para incorporar mejor así las estructuras sintácticas occidentales.

\subsection{Las traducciones extranjerizantes de LuXun}

En las traducciones extranjerizantes de $\mathrm{Lu}$ Xun, merece destacar la de Almas muertas de Nikolai Gógol, su escritor favorito. Atendiendo a que Lu Xun dedicó tanto esfuerzo a traducir esta obra a partir de una versión en alemán, Almas muertas está considerada la traducción más importante de la última etapa de su actividad como traductor. En esta traducción encontramos muchos pasajes en los que se plasman las ideas de Lu Xun sobre la extranjerización y que recogemos a continuación. Para que sirva de referencia y poder ver con mayor facilidad las características de la traducción de Lu Xun, incluimos también los mismos pasajes en la versión del traductor Zhen Hailing, que es más moderna y opta por la apropiación en lugar de la extranjerización.

\section{Ejemplo I}

\section{Texto original en alemán:}

[...] sie trugen ihr Kopfhaar weder in Form von Büscheln, noch Locken, noch, a la Diable memporte (hol mich der teufel), wie die Franzosen es nennen. Das Haar war entweder kurz geschoren oder glatt ins Gesicht gekämmt, wie geleckt, und ihre Gesichtszüge waren rund und kräftig. (Gógol, I9o9: I4)

El pelo no lo llevaban ni recogido en un moño, ni rizado, ni a la Diable memporte, como dicen los franceses. Lo llevaban o corto o peinado de tal manera que parecía estar pegado a la cara, que era redonda y con facciones fuertes. ${ }^{3}$

3 Las traducciones del alemán al español son literales y están realizadas por Christian Olalla Soler, a quien queremos agradecer su ayuda.
Texto traducido por Lu Xun:

他们的发样既不挂落, 也不卷缩, 又不是 法国人的 a la Diable memporte ${ }^{4}$ 式, 头发 是剪短的, 或者梳得很平, 他们的脸相因 此就越加显得浑圆, 威武。(Gógol, 20II: I2)

No llevaban el pelo suelto, ni en rizos, tampoco al estilo «a la Diable m'emporte», como llamaban los franceses. Su pelo era muy corto, o liso, por eso, sus caras se presentaban más redondas y fuertes. ${ }^{5}$

\section{Texto traducido por Zhen Hailing:}

他们不喜欢留那种一撮毛式的冠式发型, 也不喜欢留鬃发, 更不愿理成法国人说的那 种 “活见鬼”发型。他们的头发要么剪得 很短, 要么梳得十分光洁, 而他们的脸庞就 越发显得滚圆、盛气凌人。(Gógol, 2005: I2)

No les gustaba llevar el pelo en mechones, ni en rizos, ni mucho menos al "estilo de encontrarse con un fantasma vivo”, como llamaban los franceses. Su pelo o era muy corto, o brillante y reluciente, por eso, sus caras parecían muy redondas y robustas.

La traducción de Lu Xun y la de Zhen Hailing son muy diferentes al verter al chino la expresión «a la Diable memporte». Lu Xun la repite sin más, añadiendo una nota a pie de página en la que explica el significado de esta frase en francés. En cambio, en la otra versión, el traductor sustituye la frase francesa por 活见鬼 buo jian gui, que es una traducción literal. El método que utiliza Lu Xun permite que los lectores chinos tengan acceso a la expresión original francesa $y$, sin ninguna duda, le proporciona el toque exótico del texto original.

4 Aquí Lu Xun pone una nota al pie de la página: 法国话, 直译是 “恶魔捉我”，意译是 “任其自 然” (palabras francesas que traducidas literalmente significan «el diablo me arresta»; si se traducen libremente, significan «dejarlo al natural»).

5 Todas las traducciones del chino al español son de las autoras. 


\section{Ejemplo 2}

Texto original en alemán:

Und wie wunderbar treffend ist alles, was aus den tiefsten Tiefen Russlands hervordringt, wo es weder deutsche, noch finnische noch irgend welche anderen Volksstämme gibt, sondern alles ein urwüchsiges Urprodukt des lebendigen wagemutig kecken russischen Geistes ist [...]. (Gógol, 1909: 175)

Qué maravilloso es encontrar todo aquello que produce las profundidades más profundas de Rusia, donde no hay alemanes, ni fineses ni las gentes de ningún otro pueblo. Ahí sólo vive el producto de la tierra, la encarnación del espíritu ruso, vivaz, atrevimiento* y fresco [...].

Texto traducido por Lu Xun:

凡从并不夹杂德国人，芬兰人，以及别的 民族，只住着纯粹，活泼，勇敢的俄罗斯 人的俄国的最深的深处所发生的言语, 都 精确的出奇。(Gógol, 20II: II5)

Las lenguas que vienen de las profundidades más profundas de Rusia, donde no viven alemanes, ni fineses, ni gente de otras naciones, excepto los puros, valientes y enérgicos rusos, son extremadamente precisas.

Texto traducido por Zhen Hailing:

来自俄罗斯民间的语言是非常精确的，因 为在俄罗斯腹地, 既没有德国人, 没有芬兰 人，也没有其他外国人，那里都是些土生土 长、生动活泼的语言大师。(Gógol, 2005: II2)

Las lenguas que vienen de la masa rusa siempre son muy precisas, porque en lo más profundo de Rusia, no viven alemanes, ni fineses, ni gente de otros países: sólo viven los nativos de allí que son maestros vivos y atrevidos de lenguas.

Estas dos traducciones tienen una diferencia muy interesante: $\mathrm{Lu}$ Xun mantiene la sintaxis occidental en su versión, insertando dentro de la oración principal una larga cláusula atributiva, mientras que Zhen cambia el orden oracional del texto original y sitúa la cláusula después de la oración principal. Además, añade la conjunción «porque», que le sirve para explicitar la lógica entre las oraciones.

Hay que reconocer que la traducción de $\mathrm{Lu}$ Xun resulta algo difícil de entender en chino. Se debe releer para dar con el sujeto, el verbo y la cláusula atributiva. Si el lector dominara alguna lengua occidental, se daría cuenta de que esta estructura «extraña» es el resultado de una conservación fiel de la sintaxis extranjera, la cual es muy diferente de la china. Con el uso de la extranjerización, Lu Xun busca presentar a los lectores chinos la sintaxis occidental tal como es e introduce elementos extranjeros a la cultura china para fomentar el desarrollo lingüístico chino.

\section{Ejemplo 3}

\section{Texto original en alemán:}

[...] überall im Leben, in seinen harten, rauhen und ärmlichen, in den unsaubern, schimmelbedeckten niederen Schichten - wie in der sauberen Korrektheit und Monotonie der höheren Stände- überall begegnet uns, wenn auch nur ein einziges Mal im Leben eine Erscheinung, die nichts gemein hat mit alledem, was wir bisher gesehen, die wenigstens einmal ein neues Gesühl in uns entzündet, das keine Ähnlichkeit mit jenen hat, die uns durch unser ganzes Leben begleiten. (Gógol, I909: I45)

En la vida, tanto si transcurre en las duras, ásperas, humildes y sucias calles de los barrios bajos — como en la pulcritud y monotonía de las casas señoriales- llega un momento, quizás solo uno, en el que al ser humano se le aparece algo que no guarda similitud con lo que ha visto hasta entonces y que despierta en él un sentimiento nuevo, totalmente contrario a aquellos que lo han acompañado a lo largo de los años. 
Texto traducido por Lu Xun:

凡在人间, 在粗笨的, 冷酷的, 穷苦的, 不干净的, 发需的下等人里一也如在干净 188 的, 规矩的, 单调的上流人们里一样一无 论在哪里, 我们总会遇到一回想来从未见过 的现象, 至少也总会有一回会燃起向来无与 相比的感情。(Gógol, 20II: 95-96)

En el mundo, tanto a la gente de clase baja que es torpe, indiferente, pobre, sucia y mohosa - como a la gente de clase alta que es limpia, conformista y aburrida- no importa donde se estén, siempre le ocurre algo que no guarda similitud con lo que ha visto hasta entonces, por lo menos siempre habrá una vez en que tengan unos sentimientos que nunca han sentido antes.

Texto traducido por Zhen Hailing:

在人的一生中, 不论他的处境如何, 不管 他是生活在粗俗贫穷、落后而又龂龊的底 层, 还是生活在单调冷酷、整洁但却乏味 的上流社会, 他总归要在人生道路上遇见 一种他以前从未遇见过的现象。这种现象 哪怕只有一次, 但它总归要在他的心中激 起一种他向来不曾感受过的感觉。(Gógol, 2005: 94)

En la vida de una persona, no importa en qué situación se encuentre, no importa si pertenece a la clase baja que es vulgar, pobre, atrasada y sucia, o a la clase alta que es monótona, fría, limpia pero aburrida, siempre se encuentra con algo que no ha experimentado nunca. Aunque sea sólo una vez: lo que provoca siempre en su corazón unos sentimientos que nunca han tenido antes.

La característica más notable de la traducción de Lu Xun consiste en que el traductor ha incorporado los signos de puntación «—» del texto original. En cambio, Zhen prescinde de este signo de puntuación, impropio del chino y añade unos conectores para indicar la lógica oracional. Con ello, su versión se presenta más fluida y resulta más fácil de entender que la de Lu Xun.

Con este breve recorrido, podemos ver que las traducciones de Lu Xun son fieles a sus ideas del método de extranjerización. Presentan las palabras exóticas con la mayor semejanza posible al original, incluso con grafías distintas a los caracteres chinos, conservan la sintaxis singular de los textos originales y mantienen los signos de puntuación extranjeros. En algunos casos, para lograr el fin de mostrar «lo extranjero» en el texto traducido, Lu Xun sacrifica la fluidez de su traducción, lo que con frecuencia aumenta la dificultad de comprensión del texto traducido.

\section{VENUTI Y SUS IDEAS SOBRE \\ LA EXTRANJERIZACIÓN}

Lawrence Venuti es un conocido teórico de la traducción estadounidense. Como americano de origen italiano, Venuti presta mucha atención a la traducción de las obras de lenguas consideradas minoritarias en la sociedad angloamericana. Desde finales de la década de i980 ha publicado varios libros en los que plasma sus pensamientos a propósito de los métodos de extranjerización y apropiación, como The Translator's Invisibility -A history of translation (1995) o The Scandals of Translation: Towards an Ethics of Difference (I998). Está considerado como una de las figuras más influyentes y controvertidas en los círculos de traductología actual. Igual que Lu Xun, además de teórico, Venuti es también un traductor sobresaliente, cuyos proyectos de traducción han recibido varios premios y distinciones.

\section{I. La teoría de la traducción de Venuti}

Para comprender sus ideas sobre traducción es importante familiarizarse primero con un concepto que él mismo propone: la invisibilidad 
del traductor, idea recurrente en sus libros y artículos y que constituye la base de su teoría de la traducción.

Venuti denuncia que hoy en día en la cultura angloamericana existe la tendencia de hacer invisibles a los traductores al inglés. La mayoría de las editoriales, críticos y lectores de EE.UU. consideran una traducción aceptable si la pueden leer con fluidez, es decir, cuando les produce la ilusión de no estar leyendo una traducción, sino un texto escrito originalmente en inglés. En consecuencia, se encubre la intervención crucial del traductor, al que se invisibiliza sistemáticamente. Según Venuti, la invisibilidad del traductor está estrechamente relacionada con el método de la apropiación y tiene varios efectos negativos. Por un lado, refuerza la hegemonía de la lengua inglesa. Venuti (1995: 15) denuncia que al acostumbrar a los lectores de lengua inglesa a este tipo de traducciones, la cultura angloamericana se torna cada día más narcisista con respecto a su propia cultura y menos receptiva a valores extranjeros, $\mathrm{y}$ al final puede terminar por no aceptar los rasgos exóticos o distintos en las traducciones. Por otro lado, la invisibilización de los traductores repercute en la mala situación económica y social de su profesión.

A ojos de Venuti (ib.: 20), la apropiación, que caracteriza la mayoría de los textos traducidos en la sociedad angloamericana, consiste en «an ethnocentric reduction of the foreign text to target language cultural values, bringing the author back home». Dicha reducción de «lo extranjero» del texto original se realiza mediante el uso de estructuras sintácticas, palabras y convenciones familiares para los lectores y provoca en ellos un efecto de transparencia. En cambio, la extranjerización «entails choosing a foreign text and developing a translation method along lines which are excluded by dominant cultural values in the target language» (Venuti, 1998: 242). Así, las traducciones extranjerizantes constituyen un medio para promover la diversidad cultural y desafiar el inglés estándar. Venuti se declara partidario del segundo método traductor, con lo cual pretende resaltar las diferencias de las culturas minoritarias frente a la sociedad angloamericana, y al tiempo mejorar la situación social y económica de los traductores.

\subsection{Las traducciones extranjerizantes de Venuti}

Como hemos apuntado ya, además de ejercer como teórico de la traducción, Venuti lo hace también como traductor. Para ilustrar sus ideas sobre la traducción, en la tabla I recogemos a modo de ejemplo su traducción de un poema del italiano Milo De Angelis.

En la traducción de este poema vemos cómo Venuti lleva a la práctica su método extranjerizante. A diferencia de lo que sucedería con la apropiación, Venuti no ha cambiado el efecto de discontinuidad del texto original, que lo haría más fácil de entender en una primera lectura. Conserva las estructuras sintácticas rotas y los saltos de línea abruptos, creando así una traducción no fluida que supone un reto a los valores estéticos angloamericanos, donde predominan los textos más «transparentes».

Dos claros ejemplos este fenómeno los encontramos en el primer y el cuarto verso. E1 primer verso en inglés empieza diciendo «came to mind», en minúscula y sin sujeto, lo cual es inusual en inglés y se puede ver como una licencia poética. El texto original carece de sujeto explícito en el primer verso, pero se ajusta perfectamente a la gramática italiana. De hecho, este sujeto coincide con el título del poema, «L' idea», al que se remite a través del participio «venuta». En la traducción, Venuti enlaza el título del poema con el primer verso, como si 
TABLA I

Texto original de De Angelis

(citado por Venuti, I995: 287)

Traducción de Venuti (ib.: 288)

190

\section{Lidea centrale}

E venuta in mente (ma per caso, per l'odore

di alcool e le bende)

questo darsi da fare premuroso

nonostante.

E ancora, davanti a tutti, si sceglieva

tra le azioni e il loro senso.

Ma per caso.

Esseri dispotici regalavano il centro

distrattamente, con una radiografia,

e in sogno padroni minacciosi

sibilanti:

«se ti togliamo ciò che non è tuo

non ti rimane niente.»

\section{The Central Idea}

came to mind (but by chance, because of the scent of alcohol and the bandages)

this careful busying of oneself notwithstanding.

And still, in front of everybody, there was choosing between the actions and their meaning.

But by chance.

Despotic beings made a gift of the center

absentmindedly, with an X-ray,

and in a dream threatening bosses

hissing:

«if we take from you what isn't yours

you'll have nothing left.» no hubiera separación alguna. Por otra parte, en el cuarto verso aparece la palabra «notwithstanding» de manera aislada, igual que lo hace en italiano su equivalente «nonostante». De nuevo, se trata de un uso cuando menos muy peculiar en inglés que dificulta la comprensión por parte del lector, ya que es él quien debe establecer las relaciones lógicas entre las ideas, puesto que las palabras y la sintaxis que emplea el traductor no lo hacen. Con el mantenimiento de la sintaxis fragmentada Venuti busca reproducir la discontinuidad del texto original en su traducción y consigue producir un texto extranjerizante con el que desafiar los valores estéticos dominantes de la sociedad angloamericana.

\section{COMPARACIÓN DEL MÉTODO DE EXTRANJERIZACIÓN DE LU XUN Y VENUTI}

A través del breve recorrido que hemos hecho por el concepto de extranjerización de estos dos teóricos, observamos que presentan notables coincidencias, pero también algunas diferencias, que analizaremos a continuación.

\section{I. Puntos de común}

\section{I.I. La posición predominante de la} apropiación en la sociedad

El método de extranjerización de Lu Xun supuso una novedad para su época, puesto que en China los traductores solían adoptar el método de la apropiación y atribuían mucha importancia a la fluidez y al refinamiento del texto traducido. Ello está estrechamente relacionado con el trasfondo histórico de aquel entonces, ya que durante varios siglos, debido a la política de puertas cerradas de la dinastía Qing, la China antigua apenas mantenía contacto con el exterior. No es hasta finales del siglo XIX, a raíz de sucesivas derrotas a manos de potencias extranjeras, cuando el imperio chino se vio obligado a abrir las puertas al mundo. Fue así cómo los lectores chinos se enfrentaron, por 
primera vez, a la literatura extranjera de gran consumo. Considerando que el público chino no estaría preparado para las novelas occidentales, cuyas técnicas, perspectivas y estructuras literarias distaban mucho de las chinas, los traductores de este período se esforzaban en domesticarlas mediante añadiduras u omisiones con el fin de acercar las traducciones al gusto de la literatura tradicional china. Así, las traducciones de maestros como Liang Qichao (梁启 超) y Lin Shu (林纾), entre otros, que optaban por el método de apropiación, gozaron de una buena acogida entre los lectores chinos. En este contexto, la propuesta de extranjerización de $\mathrm{Lu}$ Xun provocó gran controversia y fue objeto de numerosas críticas por parte de sus colegas. El opositor más destacable fue Liang Shiqiu (梁实秋), escritor, traductor y teórico literario que criticó con un toque satírico el método de extranjerización de Lu Xun en varios artículos suyos, llegando a calificar sus traducciones «de no tener vida».

Aparte de provocar intensos debates en los círculos literarios, las traducciones extranjerizantes de Lu Xun apenas tenían salida en el público chino de entonces. Según un estudio de Wu Jun (2009: 105), del primer volumen de $L a$ colección de novelas extranjeras traducidas por $\mathrm{Lu}$ Xun y su hermano Zhou Zuoren sólo se vendieron 2I ejemplares, y del segundo, 20.

Por su parte, Venuti (1995: I) afirma que a día de hoy, en la sociedad angloamericana las traducciones que optan por la apropiación suelen estar bien valoradas y gozan de buena acogida, mientras que es habitual que las traducciones extranjerizantes se vean marginadas. Según el concepto individualista de la autoría en la cultura angloamericana, solamente los textos originales pueden ser auténticos, mientras que las traducciones son imitaciones o, más bien, copias falsas (Venuti, r995: 6-7). En su opinión, la traducción precisa borrar su estatus de obra de segunda que adquiere con el método de apropiación, a través del que crea un efecto de transparencia y se presenta como original.

Además de en la práctica traductora, la apropiación ocupa también un lugar preponderante en la traductología angloamericana. Merece la pena destacar la teoría de equivalencia dinámica de Eugene Nida, la cual es, en esencia y a ojos de Venuti, un llamamiento al uso de la apropiación en las traducciones, y constituye una violencia etnocéntrica que impone la norma de transparencia sobre cualquier cultura extranjera enmascarando la disyunción entre el texto original y el texto traducido.

Así, pues, el concepto y práctica de la extranjerización en traducción de Lu Xun y Venuti van en contra de la corriente principal de su época en sus respectivos países.

\section{I.2 Resistencia a la traducción fluida}

Como consecuencia de sus posicionamientos teóricos, uno de los elementos que caracterizan tanto las traducciones de $\mathrm{Lu}$ Xun como las de Venuti es su resistencia a crear textos perfectamente adaptados a las normas y costumbres de la lengua de llegada, sin dejar entrever los rasgos del original, aún a riesgo de carecer de fluidez.

De este modo, cuando leemos las traducciones extranjerizantes de $\mathrm{Lu}$ Xun, es habitual encontrar cláusulas largas y gran cantidad de préstamos y calcos. A pesar de que esta fidelidad formal sin duda afecta la fluidez de sus traducciones y aumenta la dificultad de comprensión de los lectores, es un efecto creado a propósito, tal como él mismo expone:

Toda traducción debe perseguir dos fines: por un lado, naturalmente debe ser lo más comprensible posible; por otro, debe conservar el estilo original, aunque ello a menudo entra 
en contradicción con la comprensión, porque el lector no está acostumbrado. Puesto que el original es extranjero, es normal que resulte extraño, y para que sea más fácil de asumir, sólo se le puede cambiar la ropa; no se le debe cortar la nariz o sacarle los ojos. Yo no abogo por cortarle la nariz ni por sacarle los ojos, por lo que en algunos casos prefiero una traducción no fluida. (Lu Xun, I98od: 352$)^{6}$

Del mismo modo que para Lu Xun, traducir consistía en «cambiar la ropa del texto extranjero, sin cortarle la nariz ni sacarle los ojos», Venuti también critica los cambios u omisiones que se ven en las traducciones al inglés y que tienden a eliminar «lo extranjero» encubriendo las diferencias lingüísticas y culturales entre el texto original y el traducido. Se opone a dicho método de traducción porque considera que disfraza la verdadera equivalencia semántica entre el texto original y la traducción, que no es más que una interpretación parcial del texto original, decantada a favor de los valores de la lengua y cultura inglesas, que reduce, cuando no excluye, las mismísimas particularidades que la traducción está llamada a trasmitir (Venuti, I995: 2I). Por ello propone una estrategia de resistencia que defiende que las traducciones son distintas tanto de los textos originales como de otros textos de la misma lengua de llegada. Lo explica a través de su propia experiencia de traducir los poemas de De Angelis del italiano al inglés:

$[\mathrm{M}] \mathrm{y}$ translations aim to be faithful to the linguistic and cultural differences of the Italian texts, their characteristic discontinu-

6 Texto original: 凡是翻译, 必须兼顾着两面, 一 当然力求其易解, 一则保存着原作的丰姿, 但这保 存, 却又常常和易懂相矛盾: 看不惯了。不过它原是 洋鬼子, 当然谁也看不惯, 为比较的顺眼起见, 只能 改换他的衣裳，却不该削低他的鼻子，剜掉他的眼 睛。我是不主张削鼻剜眼的, 所以有些地方, 仍然宁 可译得不顺口。(Lu Xun, I98od: 352) ity, the neologisms, syntactical shifts, staccato rhythms. (Venuti, 1995:30I)

Para crear este efecto de discontinuidad en el texto traducido, Venuti en muchos casos omite el sujeto y el verbo, emplea palabras arcaicas y recalca la brusquedad de los saltos de línea. Así se opone a la norma de fluidez de la cultura angloamericana y obliga al lector a cambiar sus expectativas de leer traducciones fluidas.

\section{I.3. La elección de los textos para traducir}

Otro elemento que comparten es la aplicación del criterio de extranjerización en la elección de obras de países «exóticos» y de géneros «desconocidos».

Según Lu Xun, el método de extranjerización no tiene que ver únicamente con la estrategia traductora, sino también con la selección de los textos objeto de traducción, lo cual se refleja en primer lugar en su preferencia por traducir obras de países considerados más «exóticos». En la época de Lu Xun había ya muchas traducciones de obras inglesas, estadounidenses, alemanas y francesas, como las realizadas por Lin Shu, Yan Fu o Liang Qichao. Sin embargo, era bastante difícil encontrar en el mercado chino traducciones de obras polacas, checas y de otros países europeos. En consecuencia, los chinos tenían escasos conocimientos relativos a estos países y cuando se hablaba de obras extranjeras, muchos pensaban sólo en las grandes potencias occidentales. Por ello, era muy necesario cambiar el concepto parcial que tenía la sociedad china sobre el mundo occidental. Según Lu Xun, como la traducción constituía la mejor representación de lo que en China se entendía por cultura occidental, era clave que los traductores adoptaran el concepto de extranjerización también al elegir textos que serían objeto de traducción y buscaran obras más «exóticas» que 
las inglesas, francesas o alemanas. Por eso, al elegir obras para traducir, Lu Xun no sólo prestó atención a obras de las grandes potencias, sino que también dio mucha importancia a obras de países poco conocidos en China. Al recordar su experiencia de traducir obras extranjeras junto con Lu Xun, su hermano Zhou Zuoren (2002: I65) expuso que solían traducir obras de países poco conocidos para el público chino y preferían en primer lugar obras de Rusia, Polonia, Chequia, Serbia, Bulgaria, Bosnia, Finlandia, Hungría, Rumanía y Grecia; en segundo lugar, de Dinamarca, Noruega, Suecia y los Países Bajos; y en tercer lugar, de Italia y España, aunque ello supusiera hacerlo a través de lenguas intermedias que ellos conocieran y no traducir directamente de los originales.

El criterio de extranjerización de Lu Xun también se manifiesta en su elección de obras de géneros literarios considerados marginales. Abogaba por traducir obras de temáticas, perspectivas y técnicas muy diferentes de las chinas, como por ejemplo las novelas de ciencia ficción y la literatura infantil. Así, Lu Xun tradujo varias novelas de ciencia ficción siguiendo la estrategia de extranjerización, entre las cuales destacan dos de Julio Verne: 《月界旅行》(Yuejie lüxing) y《地底旅行》(Didi lüxing), De la tierra a la luna y Viaje al centro de la tierra, respectivamente.

Igual que ocurría con las novelas de ciencia ficción, a principios del siglo XIX la literatura infantil era también un género literario nuevo para el público chino. Según Zhou Zuoren (2002: 32), China nunca había tenido una comprensión cabal de la literatura infantil, por lo que Lu Xun prestó mucha atención a este género literario y tradujo bastantes novelas infantiles, incluyendo 《小约翰》 (Xiao Yuehan) $[D e$ Kleine Johannes] de Frederik van Eeden, 《小 彼得》(Xiao Bide) [Was Peterchens Freunde erzäblen] de Hermynia Zur Mühlen o 《俄罗
斯童话》 (Eluosi tonghua) [Cuentos rusos] de Máximo Gorki, entre otros.

Del mismo modo, Venuti es partidario de elegir textos de temática poco conocida para la cultura angloamericana, lo que según él constituye la primera oportunidad para hacer una traducción extranjerizante. $\mathrm{Al}$ restaurar las obras «excluidas» por la cultura receptora, el traductor puede poner resistenciaa los valores vigentes de la sociedad angloamericana y, posiblemente, reformar la imagen de la cultura extranjera a ojos de los lectores. Según Venuti,

$[t]$ he selection of a foreign text for translation and the invention of a discursive strategy to translate should be grounded on a critical assessment of the target-language culture, its hierarchies and exclusions, its relations to cultural others worldwide. Before a foreign text is chosen, translators must scrutinize the current situation - the canon of foreign literatures in English, as well as the canon of British and American literature, set against patterns of cross-cultural exchange and geopolitical relations [...]. (Venuti, 1995: 309-310)

En resumen, sostiene que el método de extranjerización se refleja en dos aspectos: primero, la transmisión de «lo extranjero» del texto original en la versión traducida, es decir, el problema de «cómo se traduce»; y segundo, la selección de los temas rechazados por los valores vigentes de la cultura receptora, o, mejor dicho, la cuestión de «qué se traduce». Si el traductor cumple uno de estos dos objetivos, ya está usando el método de extranjerización.

\section{I.4. Receptores de las traducciones extranjerizantes: las élites}

Finalmente, el público al que se dirigen sus traducciones es otro de los puntos en común entre estos dos teóricos, que comparten una visión elitista de sus lectores potenciales. 
Al referirse a los lectores de traducciones, Lu Xun los divide en tres categorías: a) las élites educadas; b) los que están más o menos alfabetizados; y c) los analfabetos y, por lo tanto, excluidos de la lectura de literatura. Además señala que las traducciones tienen que ser distintas para cada grupo de lectores.

A los lectores de la categoría B, no podemos proporcionarles traducciones extranjerizantes, les deberíamos dar adaptaciones o, mejor aún, recreaciones y éstas deben ser gratificantes y que les animen a leer, no sólo que satisfagan su apetito. En cuanto a las traducciones destinadas a los lectores de la categoría A, sean las que sean, prefiero que sean fieles al texto original aún a costa del refinamiento. ( $\mathrm{Lu}$ Xun, I98ob: $38 \mathrm{I}-382)^{7}$

Según Lu Xun (I98ob: 382), las traducciones extranjerizantes no son tan fáciles como tomar un té o comer un plato de arroz, los lectores tienen que «masticar» con fuerza para entender lo que transmite el texto traducido, de modo que este tipo de traducciones sólo son aptas para las élites. La razón es sencilla: como los lectores de la categoría A posiblemente ya tienen más o menos contactos con culturas extranjeras, pueden entender mejor las traducciones extranjerizantes y tener una mayor capacidad de aceptar los rasgos exóticos en los textos traducidos, esto es, nuevos vocablos, conceptos y opiniones, así como la sintaxis de las lenguas occidentales.

Por su parte, Venuti también está de acuerdo con destinar las traducciones extranjerizantes a los lectores bien educados. Para él, estas traducciones no sirven para los lectores comunes y corrientes, a los cuales les van mejor las

7 Texto original: 供给乙的, 还不能用翻译, 至少 是改作, 最好还是创作, 而这创作又必须并不只在配 合读者的胃口, 讨好了, 读得多就够。至于供给甲类 读者的译本, 无论是什么, 我是至今主张“宁信而不顺 的”。(Lu Xun, r98ob: 38I-382) traducciones fluidas y fáciles de entender. En cambio, los rasgos exóticos que presentan las traducciones extranjerizantes suelen ser bien entendidos y aceptados por las élites. La misión del traductor consiste en conservar las diferencias lingüísticas y culturales entre el texto original y el traducido, pero estas diferencias sólo pueden ser percibidas por un grupo limitado de lectores, es decir, la élite bien educada (Venuti, I995: IOI).

En este sentido, Venuti está de acuerdo con las opiniones del famoso teórico y traductor alemán Schleiermacher, según el cual solamente los lectores expertos y conocedores de lenguas extranjeras, es decir, las élites, son capaces de entender usos distintos y especiales de la lengua. Por eso, cuando leen las traducciones, pueden captar las diferencias lingüísticas y culturales entre el texto original y el texto traducido, y disfrutar de la belleza de las lenguas. Piensa que con la capacidad de captar fácilmente los elementos nuevos en las traducciones extranjerizantes, la élite acepta mejor los factores exóticos del texto traducido, los usa en sus actividades culturales y, de esta forma, pueden influir en gran medida en la formación cultural de su país: «an educated elite controls the formation of a national culture by refining its language through foreignizing translations» (Venuti, 1995: IO2).

\subsection{Diferencias}

Además de todos los puntos en común que hemos observado entre las ideas de Lu Xun y Venuti respecto a la extranjerización, también presentan diferencias, que se resumen en sus puntos de partida y su opinión acerca del estatus del traductor.

\subsection{Puntos de partida}

La primera diferencia es su punto de partida. Mientras que Lu Xun parte de su deseo de 
apertura al extranjero para desarrollar su país y su cultura, que siente que está debilitada debido a las circunstancias sociopolíticas, Venuti busca contener el narcisismo cultural que considera que padecen los EE.UU. a partir de la apertura al extranjero de una cultura fuerte.

Lu Xun encarnaba una cultura antiquísima pero venida a menos, que acababa de abrir sus fronteras al extranjero y que necesitaba importar elementos foráneos para compensar sus propias debilidades. Por eso, sus pensamientos sobre la traducción tienen como punto de partida la conciencia de provenir de un país que se enfrenta en desventaja a la difusión cultural de las potencias extranjeras y que se esfuerza por conseguir el desarrollo del propio país a través de la traducción. Para él, la extranjerización constituía un buen método para contribuir al desarrollo tanto social como lingüístico de su país. Por eso, indica que el «tolerar la falta de fluidez» no es una «defensa pasiva», sino un «ataque activo» por parte de China (Lu Xun, I980b: 383 ).

A diferencia de Lu Xun, Venuti parte de la cultura más hegemónica del mundo: la angloamericana. Sus planteamientos se centran en la traducción de lenguas con un peso relativo mucho menor que el inglés, que es actualmente la lengua predominante a escala global. Por eso, los criterios de Venuti con respecto a la traducción están más centrados en la postura de una cultura fuerte frente a otras relativamente más débiles en el mundo.

El peso que tiene la cultura angloamericana puede verse en muchos aspectos y uno de los más evidentes es el hecho de que el inglés es la lengua más traducida en el mundo. Según Venuti (I995: I4), después de la Segunda Guerra Mundial, el inglés ha sido la lengua más traducida a otros idiomas y los libros escritos en otras lenguas no han sido traducidos al inglés en las mismas proporciones. La desigualdad entre el estatus del inglés y otros idiomas ha derivado en la expansión de la cultura angloamericana por todo el mundo, en detrimento de otras culturas, que van perdiendo peso en la sociedad de habla inglesa. Venuti quiere revertir esta situación y utilizar la extranjerización para combatir la hegemonía y la embriaguez culturales de la sociedad angloamericana.

\subsubsection{Opinión sobre el estatus de los} traductores

El segundo aspecto que diferencia estos dos autores es el estatus que otorgan a los traductores. Mientras que Venuti reivindica el papel y el estatus del traductor, Lu Xun considera al traductor un Prometeo, quien asume la misión de robar el fuego desde el extranjero y está obligado a soportar los posibles castigos que de ello se puedan derivar.

Solemos comparar a los revolucionarios con Prometeo, que roba el fuego para los hombres. Aunque al final es castigado por Dios, no se arrepiente de su comportamiento. Yo, como traductor, soy como Prometeo, pero robo el fuego desde el extranjero con el fin de cocinar mi propia carne. Si ésta tiene buen sabor o si las masas pueden obtener provecho masticando mi carne, el sacrificio de mi cuerpo no será en vano. ${ }^{8}$

Para Lu Xun lo más importante no es el afán de mejorar la situación social y económica del traductor, sino presentar a sus compatriotas las ideas extranjeras avanzadas que ayuden a China a salir del subdesarrollo. Si el traductor consigue

8 Texto original: 人往往以神话中的Prometheus比 革命者, 以为窃火给人, 虽遭天帝之虐待不悔, 其博 大坚忍正相同。但我从别国窃得火来, 本意却在煮自 己的肉的, 以为倘能味道较好, 庶几在咀嚼者那一 面也得到较多的好处, 我也不枉费的身躯。(Lu Xun, I98ob: 209) 
«robar el fuego» del extranjero, también tiene que aceptar los posibles castigos derivados de su acción. De ello se deduce, pues, que al introducir ideas extranjeras con gran exactitud, deberían prepararse para recibir posibles críticas por parte de los lectores, ya que tal vez sus traducciones les resultarán difíciles de entender o divergirán de las costumbres de la cultura tradicional china. Por ello, es imprescindible que el traductor tenga cierto espíritu de autosacrificio.

A diferencia de Lu Xun, Venuti presta más atención al estatus del traductor y considera necesario tomar medidas para mejorar su mala situación económica y social en la cultura angloamericana.

Según Venuti, durante mucho tiempo el traductor ha padecido explotación económica y no ha disfrutado de un papel bien reconocido en la sociedad angloamericana y demuestra su opinión trayendo a colación algunas realidades:

Blackburn's difficult situation has been faced by most freelance English-language translators throughout the postwar period: below-subsistence fees force them either to translate sporadically, while working at other jobs (typically editing, writing, teaching), or to undertake multiple translation projects simultaneously, the number of which is determined by the book market and sheer physical limitations. (Venuti, I995: 9)

Para mejorar la mala situación económica, laboral y social en que se encuentra el traductor, es preciso romper la ilusión de la invisibilidad traductora y hacer visibles los traductores tanto en las traducciones como en la sociedad. Una de las medidas más eficaces para ello es utilizar el método de la extranjerización, presentando las traducciones como lo que son en realidad, lo que, sin duda alguna, contribuirá a visibilizar a los traductores, y terminará por mejorar su situación social y económica.
Uno de los motivos que posiblemente expliquen esta divergencia en cuanto al papel del traductor es que en la época de Lu Xun, ni él ni el resto de traductores chinos dependían de la traducción como único método de subsistencia, sino que era una actividad más en su labor como intelectuales. En tiempos de Venuti, en cambio, la profesión de traductor sí existe como tal aunque, como él denuncia, carece de visibilidad y, en muchos casos, de buenas condiciones laborales y es necesario reivindicarla.

\section{CONCLUSIONES}

Pese a que cuando hablamos de la extranjerización en traducción, el primer nombre que nos viene a la mente es sin duda el del traductólogo y traductor Lawrence Venuti, tal como hemos mostrado aquí, antes de que popularizara este concepto en Occidente, el también teórico y traductor chino Lu Xun planteó su propio método de extranjerización. En este artículo, hemos revisado las propuestas teóricas de ambos para ver las diferencias y los puntos en común existentes entre ellos.

Resulta interesante ver cómo, aun partiendo de distintas culturas y circunstancias históricas, los dos teóricos coincidían en sus planteamientos sobre la extranjerización: la destacan frente a la apropiación, método predilecto de sus traductores contemporáneos y que critican abiertamente; creen que el método de traducción está vinculado al tipo de texto que se procese; y tienen una visión elitista de los lectores, quienes consideran que deben contar con una alta preparación para entender los textos y poderlos divulgar. Por otro lado, también hay unas variables que diferencian los métodos de extranjerización de Lu Xun y de Venuti: si el chino traduce para educar a la gente y crear una cultura híbrida y más avanzada, el norteamericano ve en 
la traducción extranjerizante una herramienta para proteger las culturas minoritarias frente a la supremacía de la cultura hegemónica angloamericana y evitar al mismo tiempo el empobrecimiento de la cultura receptora.

Se trata de posturas teóricas bastante extremas que se deben entender en su contexto particular, ya que están marcadas por la situación sociopolítica y la cultura donde se originaron en cada caso. De hecho, los postulados tanto de $\mathrm{Lu}$ Xun como de Venuti dieron pie a fervorosos debates de carácter teórico, pero tuvieron un impacto social reducido en la práctica, puesto que muchas de sus traducciones, como hemos mostrado ya en el caso de Lu Xun, no gozaron de una gran acogida por parte del público y no representan la corriente mayoritaria en la práctica de la traducción, hecho que merecería un análisis más detallado.

Para terminar, consideramos que comparar los pensamientos de traducción de un teórico chino y de un traductólogo occidental constituye en sí mismo un tema muy interesante e innovador. Aunque la teoría sobre traducción china cuenta con una larga historia y muchos estudiosos sobresalientes, en Occidente se conoce más bien poco. Con este modesto trabajo, que ha combinado en análisis teórico contrastivo con ejemplos de aplicación práctica, pretendemos efectuar un primer estudio en este sentido y esperamos que a partir de aquí se puedan iniciar más investigaciones, analizando traductores y traductólogos chinos más actuales, que acerquen la realidad china a otras realidades de modo que no sigan coexistiendo de espaldas, como si no compartieran ideas y puntos de vista o no pudieran beneficiarse también de las discrepancias que puede haber entre ellas.

RECIBIDO: ABRIL DE 2013

ACEPTADO: JUNIO DE 2013

VERSIÓN FINAL: OCTUBRE DE 2013

\section{REFERENCIAS BIBLIOGRÁFICAS}

Gógol, N. (I909). Die Abenteuer Tschitschikows oder Die toten Seelen. Leipzig: Georg Müller. (Trad. Otto Buek).

Gógol, N. (I980). Almas muertas. Barcelona: Editorial Planeta. (Trad. José Latín Entralgo).

Gógol, N. (2005). Sigui Ling [Almas muertas]. Beijing: Zhongguo Shuji Chubanshe. (Trad. Zhen Hailing).

Gógol, N. (20II). Sigui Ling [Almas muertas]. Wuhan: Editorial Changijiang Wenyi Chubanshe (Trad. Lu Xun).

Gu, J. (顾钧) (2009). Lu Xun Fanyi Yanjiu [Estudios sobre las traducciones de Lu Xun]. Fuzhou: Fujian jiaoyu.

Guo, Y. (郭延礼) (1999). Zhongxi Wenhua Pengzhuang yu Jindai Wenxue [Desencuentros entre la cultura oriental y la occidental, y la literatura moderna] Shandong: Shandong jiaoyu chubanshe.

Lu, X. (鲁迅) (1980a). Lu Xun Quanji [Las obras completas de Lu Xun]. vol. 3. Beijing: Renmin wenxue chubanshe.

- (I98ob). Lu Xun Quanji [Las obras completas de Lu Xun].vol. 4. Beijing: Renmin wenxue chubanshe.

- (I980c). Lu Xun Quanji [Las obras completas de Lu Xun]. vol. 6. Beijing: Renmin wenxue chubanshe.

- (rg8od). Lu Xun Quanji [Las obras completas de Lu $X u n]$. vol. ro. Beijing: Renmin wenxue chubanshe.

- (I980e). Lu Xun Quanji [Las obras completas de Lu Xun]. vol. Ir. Beijing: Renmin wenxue chubanshe.

Tai, Y. (20I2). «La traducción literal del escritor Lu Xun (I88I-I936) como impulso a la lengua vernácula (baihua) y a la nueva literatura en China». Estudios de Traducción, vol. 2, pp. I57-I66.

Venuti, L. (I995). The Translator's Invisibility: A History of Translation. Londres: Routledge.

Venuti, L. (1998). The Scandals of Translation: Towards an Ethics of Difference. Londres/Nueva York: Routledge.

Wu, J. (吴钧) (2009). Lu Xun Fanyi Wenxue Yanjiu [Estudios sobre las traducciones literarias de Lu Xun]. Ji'nan: Qilu.

Zhou, Z. (周作人) (2002a). Ertong de Wenxue [Literatura infantil], en: Zhou Zuoren, Yishu yu shenghuo [Arte y vida]. Shijiazhuang: Hebei jiaoyu chubanshe.

Zhou, Z. (周作人) (2002b). Lu Xun de Qingnian Shidai [Años de juventud de Lu Xun]. Shijiazhuang: Hebei jiaoyu chubanshe. 